\title{
Evaluating E-Commerce Trust Using Fuzzy Logic
}

\author{
Farid Meziane and Samia Nefti \\ School of Computing, Science and Engineering \\ University of Salford, \\ Salford, M5 4WT, United Kingdom
}

\begin{abstract}
Trust is widely recognised as an essential factor for the continual development of Business to Customer Electronic Commerce (B2C EC). Many trust models have been developed, however, most are subjective and did not take into account the vagueness and ambiguity of EC trust and the customers' intuitions and experience when conducting online transactions. In this paper, we develop a fuzzy trust model using fuzzy reasoning to evaluate EC trust. This trust model is based on the information customers expect to find on an EC website and that is shown to increase customers trust towards online merchants. We argue that fuzzy logic is suitable for trust evaluation as it takes into account the uncertainties within E-commerce data and like human relationships, it is often expressed by linguistics terms rather then numerical values. The evaluation of the proposed model will be illustrated using two case studies and a comparison with two evaluation models was conducted to emphasise the importance of using fuzzy logic.
\end{abstract}




\section{Introduction}

Business to Consumer (B2C) Electronic Commerce (EC) has seen a phenomenal growth since the development of the internet and there is a growing interest from many organisations to use it as a way to improve their competitiveness and reach a wider customer base. In $\mathrm{B} 2 \mathrm{C} \mathrm{EC}$, the concept of trust is crucial because it affects a number of factors essential to online transactions, including security and privacy. It is widely acknowledged that without trust EC cannot reach its full potential [Cheskin, 1999]. Among the most cited concerns of EC customers are the low level of personal data security, inconvenience systems, disappointing purchases, unwillingness to provide personal details and mistrust of the technology [Shapiro et al., 1992; Mayer et al.,1995; Lewicki and Bunker, 1996; Cheskin, 1999; Matthew and Turban, 2001].

Kasiran and Meziane (2002) developed a trust model for B2C EC that is based on the kind of information customers are looking for on a vendor's website to help them decide whether to engage in a transaction or not. The model identified four major factors that need to be present on a merchant's website to increase customers' trust when shopping online. These factors are: Existence, Affiliation, Policy and Fulfilment. The information the customer needs to collect to satisfy the existence factor include physical existence such as the merchant's telephone number, fax number and postal address; mandatory registration and peoples' existence. These are known as variables. The affiliation factor looks at third party endorsement, membership and portal and the policy factor looks at information with regards to customer satisfaction policy, privacy statement and warranty policy. Finally, the fulfilment factor looks at delivery methods, methods of payment and the community comments. Hence, a total of twelve variables have been identified as summarised in Fig. 1. 


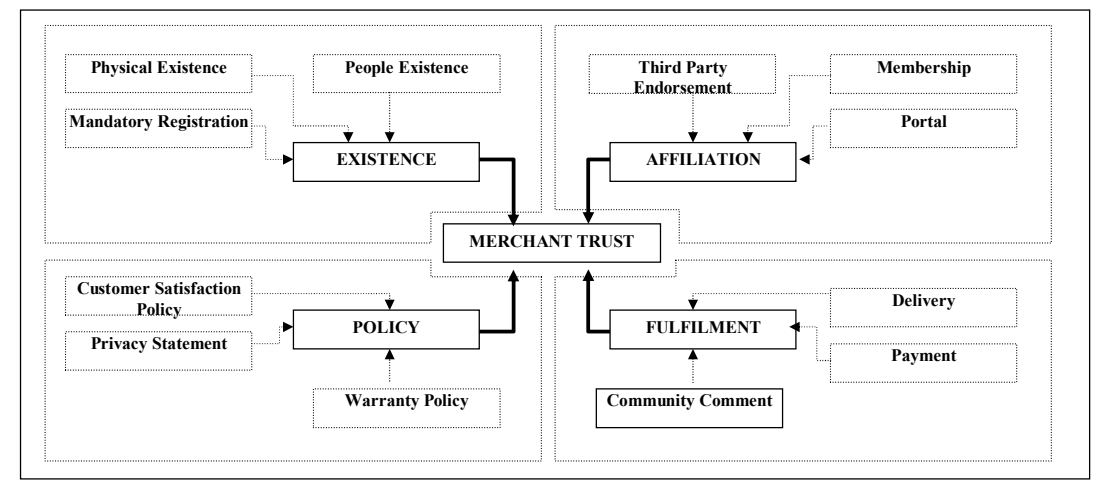

Fig. 1: The Trust Model [Kasiran and Meziane, 2002]

Given the large amount of information the model requires, an information extraction system has been developed to automate the data collection process [Meziane and Kasiran, 2003, Meziane and Kasiran, 2005]. Indeed it has been reported that users are finding it difficult to identify specific information on websites [Center for the Digital Future, 2004]. In addition we do recognize that users may not be able to make proper use of the collected information. For this purpose, we developed tools to evaluate the trustworthiness of an EC website based on the collected information. Two models have been developed in [Meziane and Kasiran, 2005] for evaluating the trust factor; the linear model and the parameterised model. More details about these two models will be provided in the comparison section (section 5).

However, for both models, we do recognise that this is not the natural way customers use to evaluate their trust towards online merchants or make the decision to buy or not. As with any other business transaction, customers develop in their mind some sort of ambiguity and uncertainties when purchasing online [Mohanty and Bhasker, 2005]. The customer may wish to classify the merchants using different preferences or take into accounts other parameters such as the cost or the brand of the product. The decision to buy or not to buy online is often based on 
user's human intuitions, common sense and experience rather then on the availability of clear, concise and accurate data [Akhter, Hobbs and Maamar, 2005]. In this paper, we develop a new trust evaluation model using fuzzy reasoning to evaluate the trust factor as it allows the encoding of the information available on the merchant's website in a form that can be used to reflect the way customers reach the decision to engage in an EC transaction.

The remaining of the paper is organised as follows. In sections 2 and 3 we describe the Fuzzy inference and fuzzy logic system and we construct the rules base in section 4 . We evaluate the newly developed fuzzy model in section 5 and we compare it with the linear and parameterised models in section 6 . In section 7 we report some related work and underline the advantages of our fuzzy system and we conclude in section 8 .

\section{The Fuzzy Inference System}

There are two concepts within fuzzy systems which play a central role in our application domain. The first one is a linguistic variable, i.e. a variable whose values are words or sentences in a natural or synthetic language. Fuzzy set theory, which is based on such paradigm, deals with the ambiguity found in semantics [Zadeh, 1965]. The second concept is that of a fuzzy IF-THEN rules in which the antecedent and the consequent parts are propositions containing linguistic variables [Mamdani, 1994]. These two concepts are effectively used in the fuzzy logic controller paradigm as shown in Fig. 2. The numerical values of the inputs $x_{i} \in U_{i}$ with $(i=1, \ldots, n)$ are fuzzified into linguistic values $F_{1}, F_{2}, \ldots, F_{n}$ where $F_{j}$ 's are defined as fuzzy sets in the input universe of discourse $U=U_{1} \times U_{2} \times \cdots \times U_{n} \subset \mathfrak{R}^{n}$. 


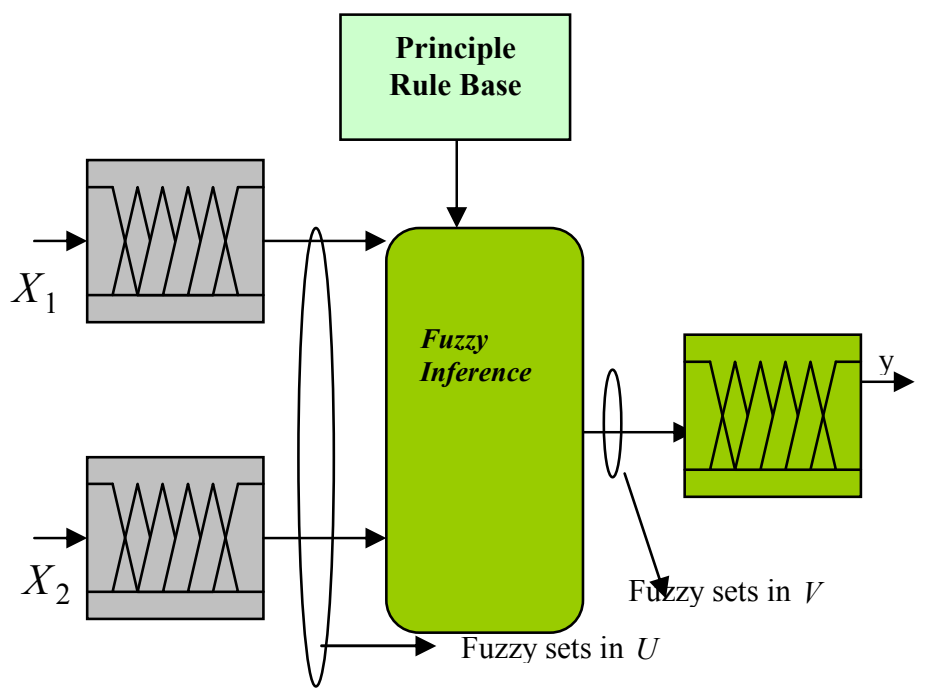

Fig. 2: The Fuzzy logic controller

A fuzzy inference engine judges and evaluates several linguistic values $G_{1}, G_{2}, \ldots, G_{n}$ in the output universe of discourse $V$ by using fuzzy IF-THEN rules which are defined in the rule base:

$R^{(j)}: I F \quad x_{i} \in F_{1}^{j}$ and...and $\quad x_{n} \in F_{n}^{j}$ Then $y \in G^{j}$

where $(j=1, \ldots M)$ and $M$ is the number of rules in the principle base. Each fuzzy IF-THEN rule in the form of (1) defines a fuzzy set $F_{1}^{j} \times F_{2}^{j} \times \ldots \times F_{n}^{j} \rightarrow G^{j}$ n the product space $U \times V$. Let $A^{\prime}$ be an arbitrary input fuzzy set in $U$. A fuzzy set $B^{m}$ in $V$ can be calculated as:

$\left.\mu_{A^{\prime} \circ R^{m}}(y)=\oplus\left(x_{1}, \ldots, x_{n}\right)^{T} \in U \mid \mu_{A^{\prime}}\left(x_{1}, \ldots, x_{n}\right) \otimes \mu_{F_{1}^{m} \times \ldots \times F_{n}^{m} \rightarrow G_{n}^{m}}\left(x_{1}, \ldots, x_{n}, y\right)\right\rfloor$

where t-norm $\otimes$ and s-norm $\oplus$ are used for the intersection and the union operations respectively. The final output is a fuzzy set in $V$, which is a combination of the $M$ fuzzy sets, $A^{\prime} \circ\left(R^{(1)}, \ldots . R^{(M)}\right)$. The membership function of this inferred fuzzy set will be:

$\mu_{A^{\prime} \circ\left(R^{(1)}, \ldots, R^{(M)}\right)}(y)=\mu_{A^{\prime} \circ R^{(1)} \oplus \ldots \oplus} \mu_{A^{\prime} \circ R^{(M)}}(y)$

The above membership function defines the fuzzy value of the output action $\mu_{B}(y)$. The crisp value of the output action can be obtained, say, by using the Centre of Gravity (COG) 
defuzzification method, where the shape of membership function, $\mu_{A^{\prime}\left(R^{(1)}, \ldots, R^{(M)}\right)}(y)$, is considered to determine the crisp value of the output action $y^{\prime}=\frac{\sum y \mu_{B}}{\sum \mu_{B}}$.

\section{Fuzzy Logic for the Trust Model}

Trust relationships among customers and vendors are hard to assess due to the uncertainties and ambiguities involved in evaluating trust in EC. For example, in the proposed trust model, the community comments variable in the fulfilment factor has a wide range of values as we may have a small or a large number of customers providing positive or negative feedback to the vendor. Hence, the number of comments and their nature will affect the decision made by the associated evaluation module. In addition, in the trust model used, there are dependencies between some variables. For example the mandatory registration variable in the existence factor is dependent on the membership and third party endorsements variables in the affiliation factor. Indeed, if an organisation is a member of an association or endorsed by a third party, we assume that this organisation is fully registered with the required authorities even though the mandatory registration was not extracted by the information extraction system.

Thus, the use of fuzzy reasoning is justified as an adequate approach to deal with evaluating trust in EC as it has the ability to quantify imprecise data and quantify uncertainties in measuring the trust factor of the vendors and to deal with variable dependencies in the system by decoupling them using human expertise in the form of linguistics rules. 
The general trust model proposed in this section is composed of five modules. Four modules will be used to quantify the trust measure of the four factors identified in our trust model (Existence, Affiliation, Policy and Fulfilment) and the fifth module will be the final decision maker to quantify the trust factor as illustrated in Fig. 3.

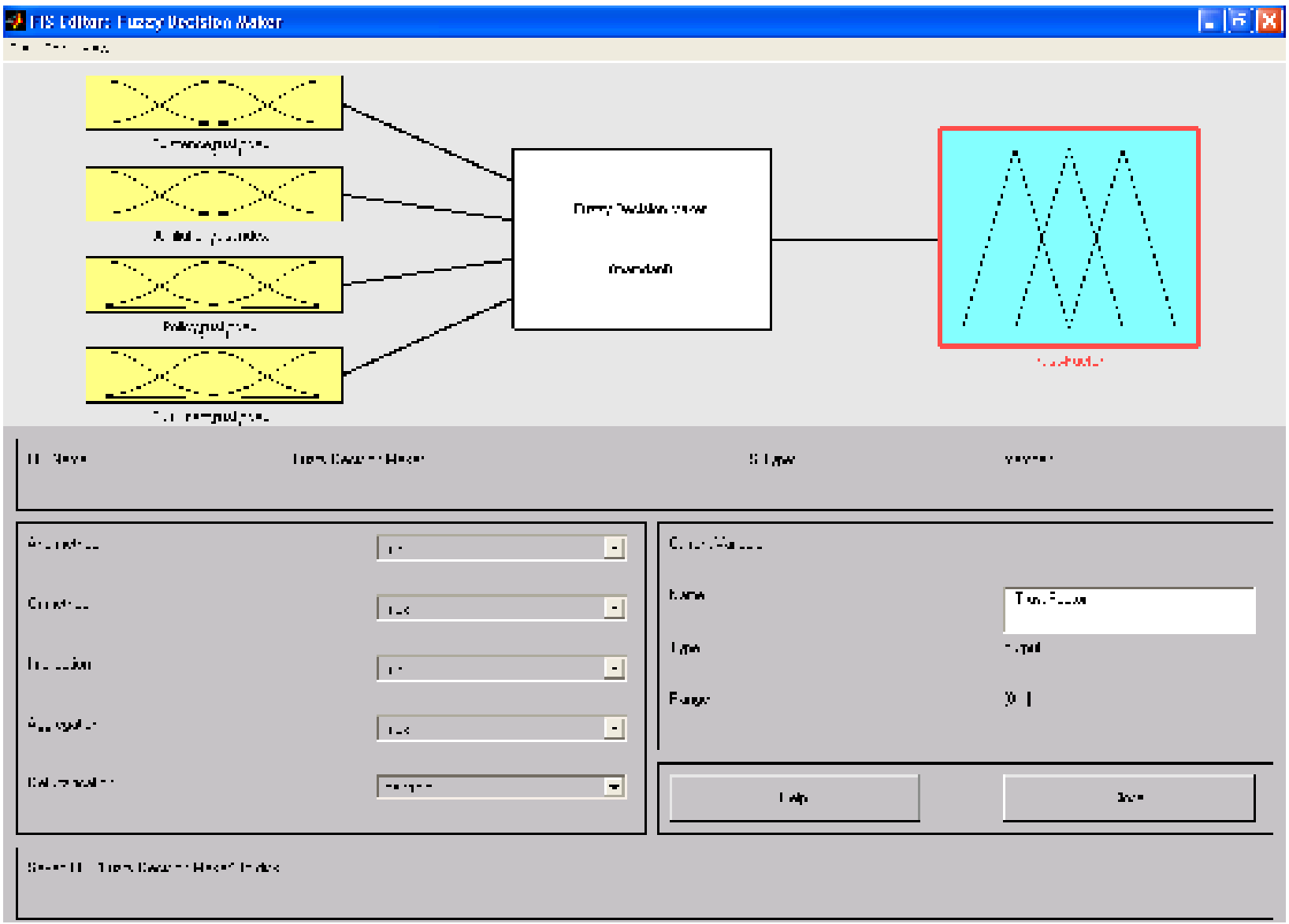

Fig. 3: The Ecommerce Fuzzy trust Model

The inputs of the Existence module are the Physical Existence, People Existence, Mandatory Registration variable and the output of the affiliation module. Indeed, as explained earlier in this section, the mandatory registration variable is dependent on the third party endorsement and membership variables of the affiliation module. We also note here that the physical existence variable is composed of three sub-variables, which are the Telephone Number, the Fax Number 
and the Physical Address. For the affiliation module, the inputs are the Third Party Endorsement, Membership and Portal variables. For the policy module, the inputs are the Customer Satisfaction, Privacy and Warranty variables. Finally, the fulfilment module has as inputs the Delivery, Payment Methods and Community Comments variables. The decision maker has as inputs the outputs of the four modules which are Existence_Trust_Index, Fulfilment_Trust_Index, Policy_Trust_Index and the Affiliation_Trust_Index. The output of this module is the trust factor of the merchant's website. In our model, this trust factor will be determined by the aggregations of the trust indices of all modules. Fig. 4 and Fig. 5 show a sample of the IF-THEN rules for the Existence and Fulfilments modules respectively.

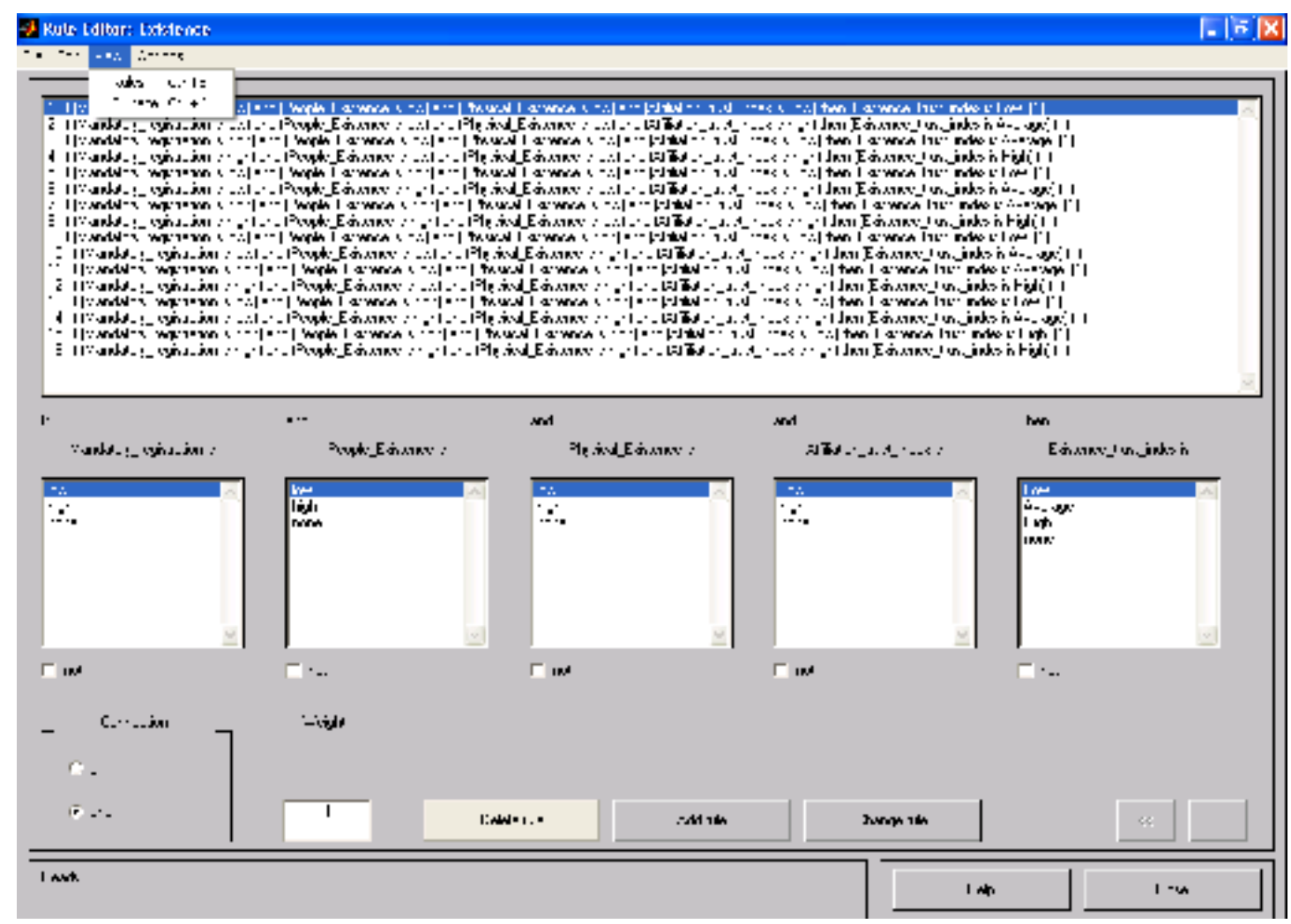

Fig.4: The Existence Module Rules 


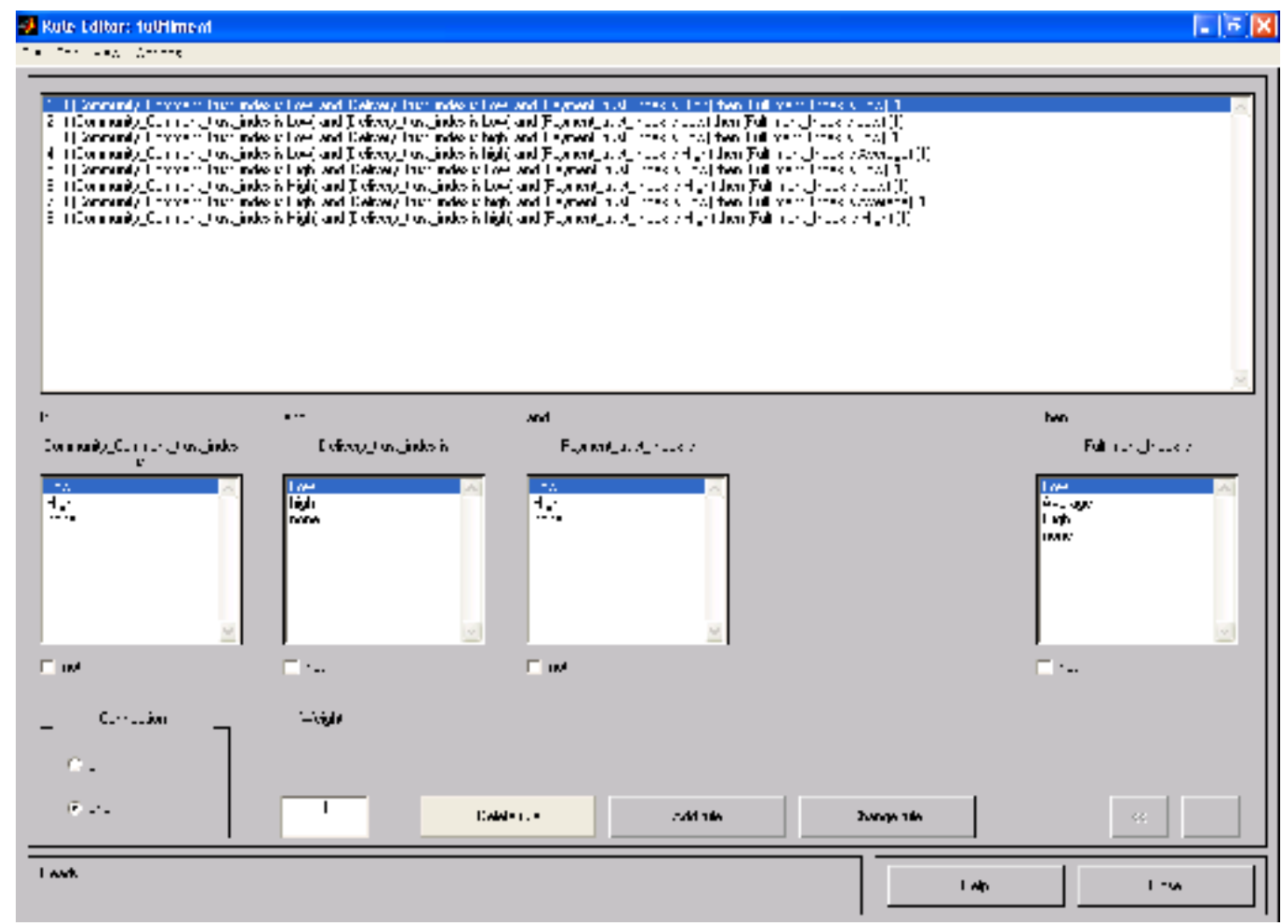

Fig. 5: The Fulfilment Module Rules

In the fuzzification phase, two membership functions described by the labels "Low" and "High" will be used for each variable related to each module. However, we introduce a third membership function "Average" for the outputs. For the decision maker module, we use all three membership functions for the inputs and output corresponding to Low, Average and High degree of trustworthiness of the website. These membership functions are represented by Gaussians defined by the centre and the standard deviation parameters. The output values are normalised within the interval $[0,1]$, with 1 for full trust and 0 for no trust. For example a vendor's website with 0.75 trust factor is considered high and should be trusted. 
The inference rules are subject to the users choice based on criteria of the risk and the gain as defined by Tao and Thoen (2001). Fuzzy inference is a process to assess the trust index in five steps: (1) Register the initial values of each variable as defined by the information extraction system. (2) Use the membership functions to generate membership degrees for each variable related to each module. (3) Apply the fuzzy rule set defined for each module onto the output space (trust index) through fuzzy 'and' and 'or' operations. (4) Aggregate the outputs from each rules related to each module, and (5) derive the trust index through a defuzzification process using the centroid method. These same steps will also be used for the decision maker module to generate the trust factor. From Fig. 6 we can see that the trust index increases with the increase of the contributing attribute of all trust indices values and decrease when the decrease of all the attribute. Fig. 7 shows a sample of the IF-THEN rules for final decision maker module.

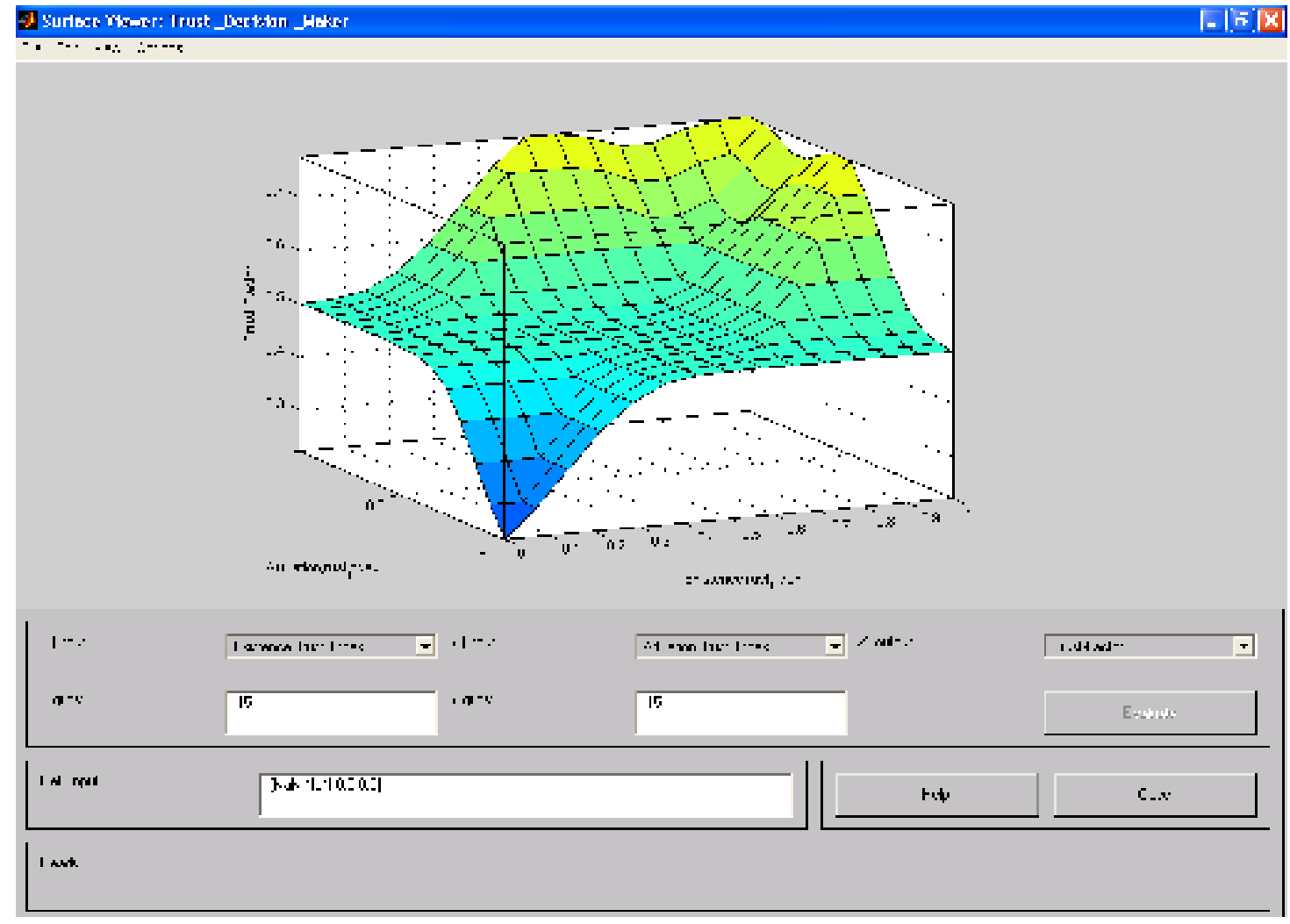

Fig. 6: Output of the Finale Decision Maker Module 


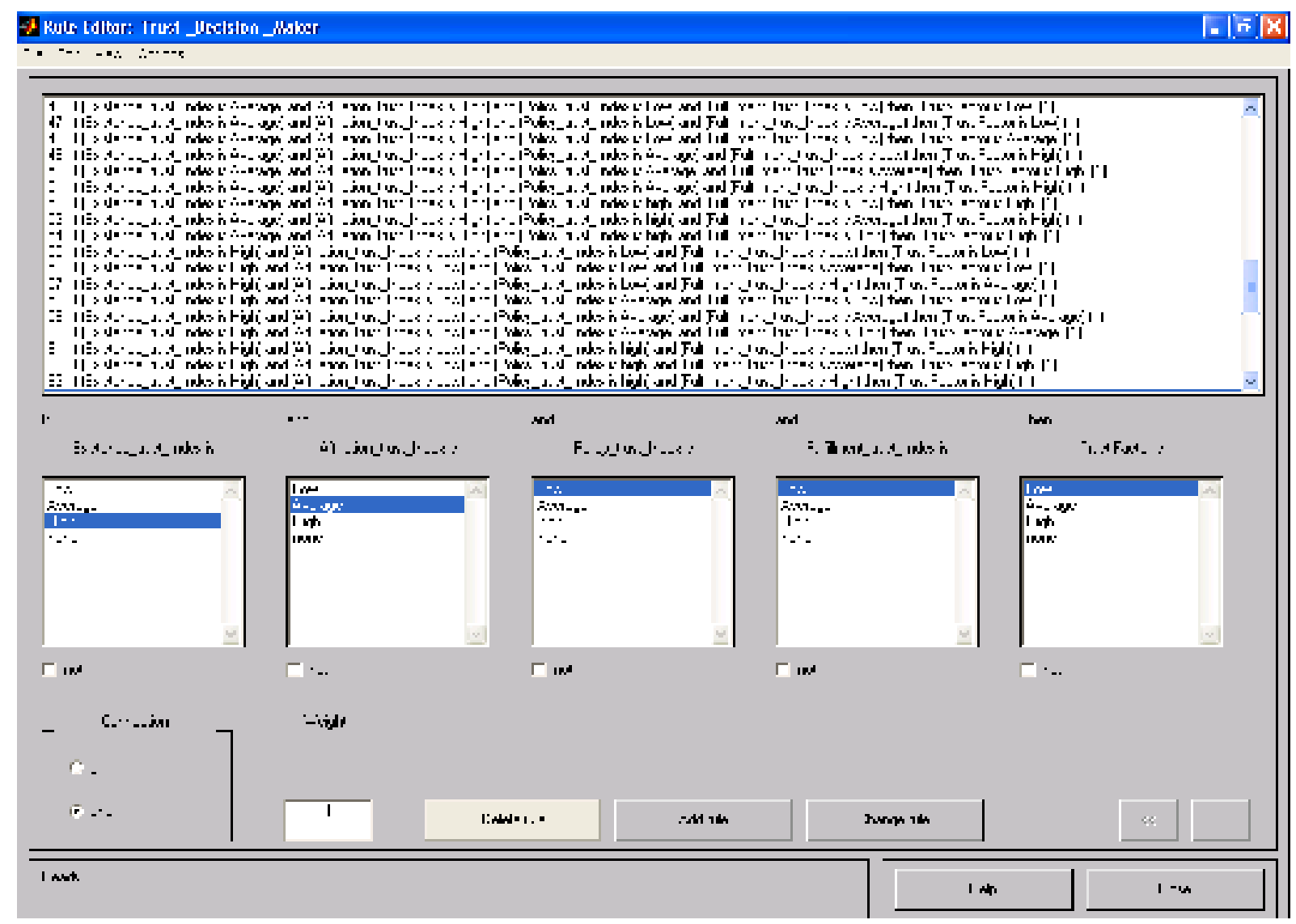

Fig. 7: The Final Decision Maker Module Rules

\section{The Construction of the Rules Base}

The decision to trust or not to trust EC as a shopping medium is up to consumers' evaluation which can be based on many factors such as price, convenience, selection of choice and the information available on the merchant's website like those defined in our model. It is widely accepted that if the economic gain is greater than the risk involved then the transaction is reasonably viable. Based on this assumption, Tao and Thoen (2001) formalised the process as: $G_{b}=P_{b} L_{b}$ where $G_{b}$ is the gain entering the EC transaction, $P_{b}$ is the risk that the consumer takes for trusting the EC merchants and $L_{b}$ is the loss the consumer has to bear when the transaction does not produce the result as expected. Consumers are usually proceeding with the 
transaction if the potential gain is greater than the potential lost and will be indifferent if both values are equal. Thus one has either to maximise the gain $G_{b}$ or minimise the risk $P_{b}$. The risk can be minimised by providing all the information required by the customer on the vendor's website. Based on this model, we assume that if a large amount of information is available on a vendor's website and if this information is valid then the vendor can be trusted. However, the importance of these factors can differ from one user to another. To validate our rules we conducted a survey through the use of an online questionnaire.

\section{System Evaluation}

To evaluate the fuzzy model developed in this paper, we have chosen two random websites. The first case study is the Denimjunkies ${ }^{1}$ site, a vintage clothing shop selling used collectable items such as jeans, jackets and shirts and the second case study is the Mesh Computers ${ }^{2}$ website, a company selling PCs and peripherals. For each factor, a set of three variables will be considered hence, a total of 13 variables (including the output of the affiliation module which is used as an input to the existence module). Thus combining all variables yield to a total of 12288 possible combinations for each website. Since we use two membership functions for all the inputs except the output from the affiliation module which has three membership functions. Given the complexity of the problem, it becomes apparent why we grouped our variables into four factors which are processed by separate modules as defined earlier. This allows us to consider only 8 possible combinations per module except the existence module which has 24 combinations. For the final decision support module, three membership functions are used Low, Average and High

\footnotetext{
${ }^{1}$ http://denimjunkies.com/

${ }^{2} \mathrm{http}: / /$ www.meshcomputers.com
} 
and this gives a possible total of 81 combinations. Table 1 summarises the information extracted from the two case studies.

Table 1:The extracted information for the case studies

\begin{tabular}{|c|c|c|c|}
\hline & Variables & http://denimjunkies.com/ & http://www.meshcomputers.com \\
\hline \multirow{3}{*}{ 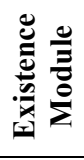 } & Physical Existence & $\mathrm{P} / \mathrm{A}$ & $\mathrm{P} / \mathrm{F} / \mathrm{A}$ \\
\hline & People Existence & yes & yes \\
\hline & Registration & yes & yes \\
\hline \multirow{3}{*}{ 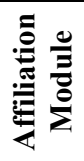 } & Endorsement & no & yes \\
\hline & Membership & yes & yes \\
\hline & Portal & no & yes \\
\hline \multirow{3}{*}{ 递 } & Customer Satisfaction & yes & yes \\
\hline & Privacy Statement & yes & no \\
\hline & Warranty Policy & no & 2 years \\
\hline \multirow{3}{*}{ 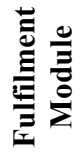 } & Delivery & immediately & delayed \\
\hline & Payment & Credit C/Debit C/Cheque & Credit C/Debit C/Cheque \\
\hline & Community Comments & no & no \\
\hline
\end{tabular}

Table 2 shows the fuzzification of the extracted values related to each variable. In the first case study, the information extraction system found two sub-variables (phone number and address) out of a possible three; hence a value of 0.6 is assigned to the physical existence variable, thus after the fuzzification step the degree of membership function of this value is 0.4 for Low and 0.6 for High. The remaining two variables (people existence and mandatory registration) in the existence factor were assigned the membership functions 1 (or High) In the second case study, all information related to the existence factor was found; thus the degree of membership function of all three existence variables will be 1 . After all the selected rules were inferred in parallel, the fuzzy operator 'and' is applied to determine the support degree of the rules. The 'and' results are aggregated and the final trust factor for case study 1 and 2 were is generated by defuzzifying the aggregation using the centroid method as shown in Fig. 8 and Fig. 9 Respectively. 
Table 2: the fuzzification of the extracted information for the case studies

\begin{tabular}{|c|c|c|c|}
\hline & Variables & http://denimjunkies.com & http://www.meshcomputers.com \\
\hline \multirow{5}{*}{ 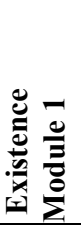 } & Physical Existence & $\{0.4 / \mathrm{L} ; 0.6 / \mathrm{H}\}$ & $\mathrm{H}$ \\
\hline & People Existence & $\mathrm{H}$ & $\mathrm{H}$ \\
\hline & Registration & $\mathrm{H}$ & $\mathrm{H}$ \\
\hline & Output 2 & $\mathrm{~L}$ & $\mathrm{H}$ \\
\hline & Output 1 & $\mathrm{H}$ & $\mathrm{H}$ \\
\hline \multirow{4}{*}{ 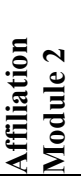 } & Endorsement & $\mathrm{L}$ & $\mathrm{H}$ \\
\hline & Membership & $\mathrm{H}$ & $\mathrm{H}$ \\
\hline & Portal & $\mathrm{L}$ & $\mathrm{H}$ \\
\hline & Output 2 & $\mathrm{~L}$ & $\mathrm{H}$ \\
\hline \multirow{4}{*}{ 串 } & Customer Satisfaction & $\{0.2 / \mathrm{L} ; 0.8 / \mathrm{H}\}$ & $\mathrm{H}$ \\
\hline & Privacy Statement & $\mathrm{H}$ & $\mathrm{L}$ \\
\hline & Warranty Policy & $\mathrm{L}$ & $\mathrm{H}$ \\
\hline & Output 3 & $\mathrm{H}$ & $\mathrm{A}$ \\
\hline \multirow{4}{*}{ 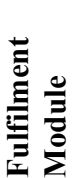 } & Delivery & $\mathrm{L}$ & $\mathrm{H}$ \\
\hline & Payment & $\mathrm{H}$ & $\mathrm{H}$ \\
\hline & Community Comments & $\mathrm{L}$ & $\mathrm{L}$ \\
\hline & Output 3 & L & A \\
\hline
\end{tabular}

Example of rules used to process the policy_trust_index for the case study 1 are as follows:

IF customer_satisfaction is Low and privacy_statement is High and warranty_policy is low THEN Policy_trust_index is Low .

IF customer_satisfaction is High and privacy_statement is High and warranty_policy is low THEN Policy_trust_index is Average.

The rules used for the aggregation of the final results for the case studies 1 and 2 and the outputs of the decision maker module are summarised in table 3.

Table 3:The trust factor of the case studies

\begin{tabular}{|l|r|r|}
\hline Outputs & http://denimjunkies.com & http://www.meshcomputers.com \\
\hline Existence_trust_index & High & High \\
\hline Affiliation_trust_index & Low & High \\
\hline Policy_trust_index & High & Average \\
\hline Fulfilment_trust_index & Low & Average \\
\hline Trust_factor & Low (0.62) & Average (0.765) \\
\hline
\end{tabular}




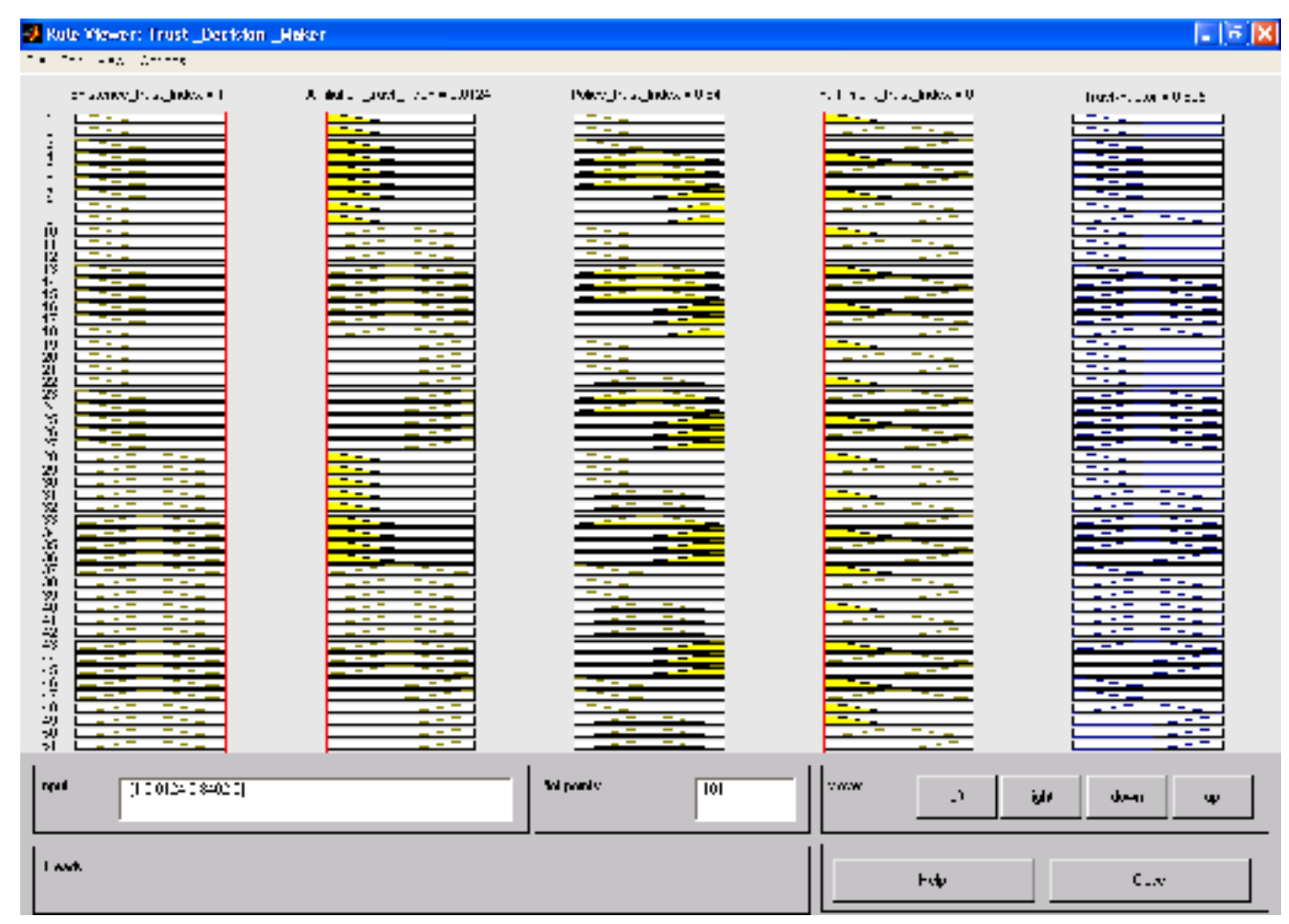

Fig. 8: Rules Aggregations and Output for Case Study 1

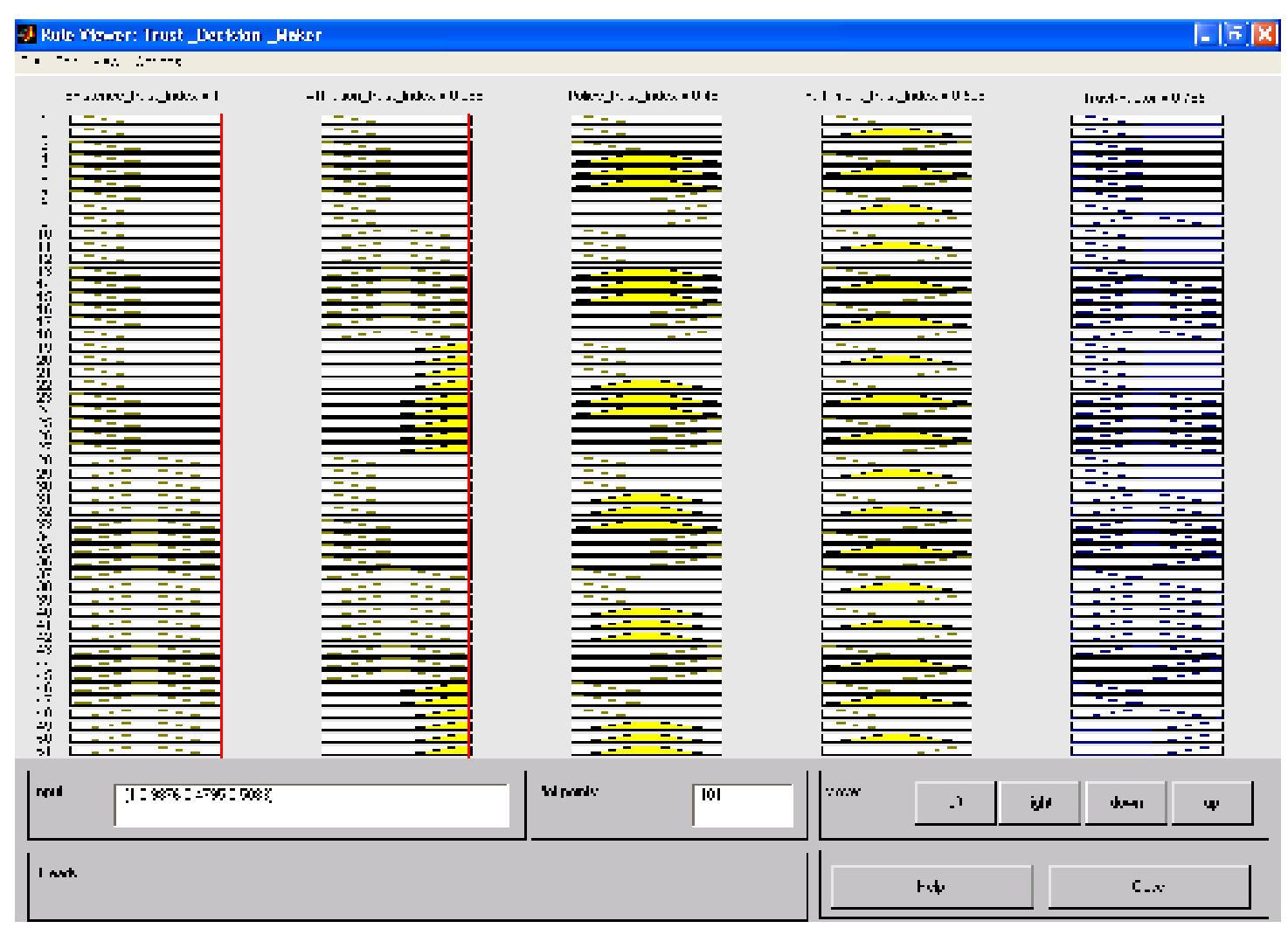

Fig. 9: Rules Aggregations and Output for Case Study 2 


\section{Comparison of the Fuzzy Model with Other Models}

Meziane and Kasiran (2005) developed two models to evaluate EC trust using the same model shown in Fig 1, the linear model and the parameterised model. Both models were based on the presence or not of the variables on the EC website. The linear model is used for new or inexperienced users. The system automatically assigns the value of 1 when a variable is found and 0 otherwise. The total is then divided by the number of variables (12) and a trust factor with a value in the interval $[0,1]$ is calculated using equation (4) were $T$ is the trust factor and $v_{i}$.represents one of the 12 variables of the trust model.

$$
T=\frac{1}{12}\left(\sum_{i=1}^{12} v_{i}\right)
$$

The parameterised model is used with more experiences users which are asked to evaluate the importance (according to their perception) of each variable by assigning the value 1 if the variable is judged important, 0.5 if the variable is fairly important and 0 if it is not important. These values are used as weights to the linear model variables and again a trust factor $T$ in the interval $[0,1]$ is calculated using equation (5).

$$
T=\frac{1}{4}\left(\frac{\sum_{I=1}^{3} E_{i} \mathcal{w}_{i}}{\sum_{i=1}^{3} \mathcal{w}_{i}}+\frac{\sum_{j=1}^{3} A_{i} \mathcal{w}_{i}}{\sum_{j=1}^{3} \mathcal{w}_{i}}+\frac{\sum_{k=1}^{3} P_{i} \mathcal{W}_{i}}{\sum_{k=1}^{3} \mathcal{w}_{i}}+\frac{\sum_{l=1}^{3} F_{i} \mathcal{w}_{i}}{\sum_{l=1}^{3} \mathcal{W}_{i}}\right)
$$

In both cases, the closer to 1 the trust factor is the higher the trust is towards the merchant's website. We have compared the results obtained by the Fuzzy model with those obtained by the linear and parameterised model and the results are shown in Table 4. The weights used for the parameterised model used in this experiment, chosen by the authors, are as follows: Physical 
existence (1), People existence (0), Mandatory registration (1), Third Party endorsement (1), Membership (0.5), Portal (0.5), Customer Satisfaction (1), Privacy statement (1), Warranty (1), Delivery (0.5), Payment (0), Community comments (0.5).

Table 4: Trust Models Comparison

\begin{tabular}{|l|r|r|r|}
\hline & \multicolumn{4}{|c|}{ Fuzzy Model } & Linear Model & Parameterised Model \\
\hline Case study 1 & 0.62 & 0.66 & 0.60 \\
\hline Case study 2 & 0.765 & 0.83 & 0.79 \\
\hline
\end{tabular}

The results obtained by the linear model, which are those provided for a new or inexperienced user, are high compared to those obtained by the other two models. The linear model results are only based on the existence or non existence of the variables on the merchant's website and this may be misleading as not all variables are of the same importance. The results of the fuzzy and parameterised models are close as in this particular experiment, those who choose the weights for the parameterised model, the authors, are themselves experts and have experience in EC transactions and hence the results are similar to those produced by the fuzzy systems were experts have produced the IF-THEN rules. By developing the fuzzy model, there is now no need for the use of the parameterised model as the expertise, on a general scale rather then chosen by individual users, is incorporated in the evaluation process. Users experience is also subjective and the use of the fuzzy model will provide a stronger and a more objective tool to all users regardless of their expertise and experience.

\section{Related Work}

Akhter et al., (2005) developed a Fuzzy logic based system for assessing the level of trust in B2C EC. In their model the trust (T) is composed of three variables which are security (S), familiarity (F) and the Website's design layout (D) hence $T=f(S, F, D)$. In addition, they have also used competitiveness (C) in the evaluation of the business transaction. Hence the business transaction 
is a function of trust and Competitiveness and formulated as $L_{B 2 C}=g(T, C)$. However, in their model they are not clear on what factors they use to evaluate each of the three variables $S, F$ and $D$. They assume that users by just using a website can decide if the trust is high, average or low with regards to these three variables. Studies on the use of websites design for example to convey trust are well documented and the characteristics well defined [Basso et al., 2001, Riegelsberg and Sasse, 2001, Hu et al., 2004].

Manchala (2000) proposes a model for the measurement of trust variables and the fuzzy verification of E-Commerce transactions. He highlights the fact that trust can be determined by evaluating the factors that influence it, namely risk. He defines cost of transaction, transaction history, customer loyalty, indemnity and spending patterns as the trust variables. Each variable is measured using semantic labels. His notation is focused on defining when two trust variables are related by an EC Trust Relationship (ECTR). Using this ECTR, a trust matrix is constructed between the two variables and a Trust Zone is established. He also describes a method for trust propagation and the construction of a single trust matrix between vendor and customer that governs the transaction. The problem with Manchala's model is that it is unclear (1) which variables should be used by default for the best results; (2) if it is actually possible for a computer to automatically establish that two variables are related by an ECTR. In his definition, he mentions a semantic relationship between the variables, but neglects to mention how this fact will be specified to the computer so that evaluation can be automated and (3) if ECTR merging will scale in the face of large trust matrices. These concerns are all related to the viability of implementing his model. These models do not support a theoretic approach to trust and they are not suitable for E-commerce [Tyron, 2003]. 
Fuzzy logic was also used for product classification in EC [Mohanty and Bhasker, 2005]. When faced with a choice of many products, users need to make a decision on which product to purchase. Taking the case of a car purchase, the authors used 5 variables which are the cost, resale value, mileage, comfort and Maintenance cost. A typical statement would be then to purchase a car with a price around a particular value, a high resale price, with a mileage around certain mileage, comfortable and a low maintenance cost. Such systems would work only on a specific type of products (cars in this case) if one wishes to purchase a Personal Computer for example, the variables would change as is the fuzzy logic system.

From a technology perspective Jøsang (1998) has shown that it is possible to develop a model for trust and that this model is a model for beliefs. In developing this model Jøsang has formulated a framework which he calls "subjective logic" which is an extension of standard logic and in part probability theory. It is the assessment mechanism that must be used to evaluate the probability associated to the information and the assessment will then be used to assist in the establishment of the requisite trust. Since the concept of trust is subjective, it creates a number of unique problems that obviates any clear mathematical result..

\section{Conclusion and Future Work}

In this paper, we presented a system based on fuzzy logic to support the evaluation and the quantification of trust in EC. Although, the system has addressed many issues that other systems did not such as taking into account the fuzzy nature of trust and using a substantial number of variables, we believe that the system can be improved in many ways. As stated in many trust models, there are other aspects that contribute to the completion of online transactions. This include the price, the rarity of the item and the experience of the customer In order to develop an 
effective decision support system, future development should include some if not all of these aspects. The price of the item is certainly an important variable as it is shown in many studies that if the price is reasonably low, customers are ready to take the highest risk to purchase the item. Online transactions also depend on customer's experience and personality. Some customers may value some variables more then others. Hence we believe that future systems should allow customers to rank trust variables according to their own perception and experience.

\section{References}

Ahuja V. (2000), Building Trust in Electronic Commerce, IT Professional, 2(3): 61-63.

Akhter F., Hobbs D. and Maamar Z. (2005), A fuzzy logic-based system for assessing the level of business-toconsumer (B2C) trust in electronic commerce, Expert Systems with Applications 28:623-628.

Ba S. (2001), Establishing Online Trust Through a Community Responsibility System, Decision Support Systems, (13):323-336.

Basso A., Goldberg D., Greenspan S. and Weimer D. (2001), First impressions: emotional and cognitive factors underlying judgments of trust e-commerce, Proceedings of the $3^{\text {rd }}$ ACM conference on Electronic Commerce, p.137143, Tampa, Florida, USA

Cheskin Research group (1999), eCommerce Study Trust, http://www.studioarchetype.com/cheskin/assets/images/etrust.pdf, 1999.

Guo J. and Sun C. (2004), Global Electronic Markets and Global Traditional Markets, Electronic Markets 14(1):412. 
Han K.S. and Noh M.H. (1999), Critical Failure Factors that Discourage the Growth of Electronic Commerce, International Journal of Electronic Commerce, 4(2):25-43.

Hu J., Shima K., Oehlmann R., Zhao J., Takemura Y. and Matsumoto K. (2004), An empirical study of audience impressions of B2C web pages in Japan, China and the UK, Electronic Commerce Research and Applications, (3):176-189.

Jøsang A. (1998), Modelling Trust in Information Society, PhD Thesis, Department of Telematics, Norwegian University of Science and Technology, Trondheim, Norway.

Kasiran M.K. and Meziane F. (2002), An Information Framework for a Merchant Trust Agent in Electronic Commerce, In Yin H., Allinson N., Freeman R., Keane J. and Hubbard S. (Eds), Intelligent Data Engineering and Automated Learning, LNCS 2412, pp 243-248, Springer.

Kasiran M.K. and Meziane F. (2004), The Usage of Third Party Endorsement in Ecommerce Websites, $7^{\text {th }}$ International Conference on Work with Computing Systems (WWCS2004), Kuala Lumpur, Malaysia, pp. 794-798.

Lewicki R.J. and Bunker B.B. (1996), Developing and Maintaining Trust in Working Relationships, In Kramer R.M. and Tyler T. (Eds.), Trust in organizations, Thousand Oaks, CA: Sage, pp. 114-139.

Mamdani E. (1994), Application of Fuzzy Algorithms of Simple Dynamic Plants, Proceedings of IEEE, 121, 585588.

Manchala D.W. (2000), E-Commerce Trust Metrics and Models, IEEE Internet Computing, March-April, pp.36-44

Matthew K.O. and Turban E. (2001), A Trust Model for Consumer Internet Shopping, International Journal of Electronic Commerce, 6(1):75-91. 
Mayer R.C., Davis J.H. and Schoorman F.D. (1995), An Integrative Model of Organizational Trust, Academy of Management Review, 20(3):709-734.

Meziane F. and Kasiran M.K (2003), Extracting Unstructured Information From the WWW to Support Merchant Existence in E-Commerce, in Dusterhoft A. and Thalheim B. (Eds), Lecture Notes in Informatics, Natural Language Processing and Information Systems, pp.175-185, GI-Edition, Bonn, Germany.

Meziane F. and Kasiran M.K. (2005), Strategizing Consumer Logistic Requirements In Ecommerce Transactions: Evaluation of Current Implementations, Proceedings of the $3^{\text {rd }}$ European Conference on Intelligent Management Systems in Operations, Salford, Manchester, UK, June 2005, pp.116-125, The Operational Research Society.

National Fraud Information Centre, http://www.fraud.org/2002intstats.htm

Riegelsberger J. and Sasse M.A. (2001), Trustbuilders and trustbusters: The role of trust cues in interfaces to ecommerce applications, Proceedings of the $1^{\text {st }}$ IFIP Conference on e-commerce, e-business, e-government, pp. 17-30, Kluwer.

Shapiro D., Sheppard B.H. and Cheraskin L. (1992), Business on a Handshake, The Negotiation Journal, pp. 365378 , October.

Tao-Huan T. and Theon W. (2001), Towards a Generic Model of Trust in Electronic Commerce, International Journal of Electronic Commerce, 5(2):61-74.

Tyrone W. A. Grandison (2003), Trust Management for Internet Applications, PhD Thesis, Imperial College of Science, Technology and Medicine University of London, Department of Computing.

Zadeh L. (1965), Fuzzy Sets, Information and Control 8, pp. 338-353. 